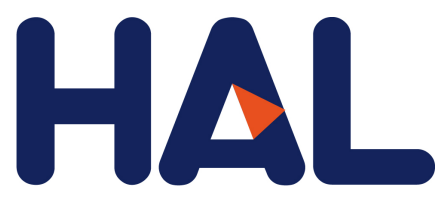

archives-ouvertes

\title{
Observer design for a nonlinear diffusion system based on the Kirchhoff transformation
}

\author{
Dyhia Benameur, Ahmed Maidi, Said Djennoune, Jean-Pierre Corriou
}

\section{To cite this version:}

Dyhia Benameur, Ahmed Maidi, Said Djennoune, Jean-Pierre Corriou. Observer design for a nonlinear diffusion system based on the Kirchhoff transformation. International Journal of Dynamics and Control, Springer Berlin Heidelberg, 2018, 6 (1), pp.154-166. 10.1007/s40435-017-0313-y . hal02130572

\section{HAL Id: hal-02130572 \\ https://hal.archives-ouvertes.fr/hal-02130572}

Submitted on 27 Nov 2019

HAL is a multi-disciplinary open access archive for the deposit and dissemination of scientific research documents, whether they are published or not. The documents may come from teaching and research institutions in France or abroad, or from public or private research centers.
L'archive ouverte pluridisciplinaire HAL, est destinée au dépôt et à la diffusion de documents scientifiques de niveau recherche, publiés ou non, émanant des établissements d'enseignement et de recherche français ou étrangers, des laboratoires publics ou privés. 


\title{
Observer Design for a Nonlinear Diffusion System Based on the Kirchhoff Transformation
}

\author{
Dyhia Benameur , Ahmed Maidi , Saïd Djennoune , Jean-Pierre Corriou
}

\begin{abstract}
This paper deals with the state estimation for a nonlinear diffusion system. An observer that reconstructs the whole state, from the available measurements, is proposed based on an equivalent linear diffusion model obtained using the Kirchhoff tangent transformation. This bijective mapping allows to apply the available and powerful state estimation theory of linear distributed parameter systems and simplifies the observer design. Hence, an observer can be designed for the obtained equivalent linear diffusion system and by using the Kirchhoff transformation, the whole state of the original nonlinear diffusion system is recovered. The observability analysis of the nonlinear diffusion system and the convergence of the proposed observer are also investigated based on the equivalent linear diffusion system. The effectiveness of the proposed observer is shown, through numerical simulation runs, in the case of a heated steel rod by considering both an uniformly distributed and a punctual boundary sensing.
\end{abstract}

Keywords distributed parameter system - nonlinear diffusion - partial differential equation - Kirchhoff tangent transformation $\cdot$ state estimation $\cdot$ observer.

\section{Introduction}

One of the important issues in control theory of distributed parameter systems (DPSs) is the state estimation, that is, the design of an observer that provides the whole state from the available measurements $[24,4$, $30,8]$. DPSs, modeled by partial differential equations (PDEs), are characterized by an infinite dimensional state [6]. Hence, the design of controllers for DPSs, following either the early or late lumping approach [24], leads to control laws of infinite dimensional nature [4].
Consequently, for implementation purposes, the whole state must be available. In practice, due to both technical and economical issues, sensing all the state variables of a distributed parameter system (DPS) is impossible. Therefore, state estimation or design of an observer for DPS is a challenging field $[12,19,5,34,23]$. State estimation consists in reconstructing the whole state of the system from limited available measurements using its dynamical model.

Generally, there are two design approaches for an infinite dimensional observer [30]. The first approach is termed early lumping and consists in reducing the DPS to a lumped parameter system (LPS), given by a set of ordinary differential equations (ODEs), using reduction techniques [15]. The objective is to take advantage of the well developed state estimation theory of lumped parameter systems (LPSs), that is, to use conventional observers, such as a Luenberger observer and Kalman filters. This approach is generally limited because the reduction process often masks the distributed nature of the system $[24,4,25]$ and may lead to erroneous conclusions about the system fundamental control theoretical properties particularly its observability $[24,25]$ which is a primary issue for the observer design. In the second approach, termed late lumping, the PDEs model is directly used both in the observability analysis and in the observer design. This allows to keep the distributed nature of the system and enhance the performance $[24,4]$. However, for nonlinear DPSs, manipulating the PDEs model is a difficult task [16].

State estimation theory for DPSs following the late lumping approach is an active research area with many open questions. This theory is well established in the case of linear DPSs [12] thanks to the semigroup theory [10] that allows to generalize many theoretical concepts developed for linear LPSs to linear DPSs [6]. For nonlinear DPSs, state estimation is an acute problem as the 
presence of strong nonlinearities $[30,1]$ that capture important phenomena like diffusion makes the observability analysis, the observer design and the convergence analysis much more difficult. This explains the few contributions reported in the literature $[2,13,17,18,32]$.

In this paper, following the late lumping approach, a state estimation strategy is developed for DPSs modeled by a nonlinear diffusion equation. This important class of parabolic PDEs describes nonlinear dynamical behaviors such as heat and mass transfer, continuous casting, filtration and phase transition, to mention a few $[11,35]$. Thus, to take advantage from the well developed observability analysis and the state estimation theories of linear DPS [6], it is proposed to derive an equivalent linear diffusion model using the Kirchhoff tangent transformation [28,3,21]. Then, a linear observer is designed, in the framework of late lumping approach, for the resulting equivalent linear diffusion model. The whole state of the original nonlinear diffusion system is reconstructed, using the Kirchhoff transformation, from the estimated state of the equivalent linear diffusion model provided by the designed linear observer. The convergence of the proposed state estimation strategy is demonstrated based on the bijective property of the Kirchhoff tangent transformation. The example of a steel rod is considered to show the effectiveness of the proposed state estimation strategy in retrieving the whole state from either uniformly distributed noisy measurements or a punctual boundary measurement.

Note that reducing the nonlinear diffusion system to a linear one allows also to overcome the intractable problem of observability analysis, that is, the location of the measurements sensors for the nonlinear diffusion system $[30,7,33,31]$. Therefore, the appropriate sensor configuration for the nonlinear diffusion system can be deduced from the observability analysis of the resulting equivalent linear model using the available powerful tools $[24,6]$.

The rest of the paper is organized as follows: In Section 2, the state estimation problem for the nonlinear diffusion system is formulated. Section 3 is devoted to the Kirchhoff tangent transformation used to linearize the nonlinear diffusion system. Section 4 gives a brief review of the observer design for linear DPSs following the late lumping approach. Section 5 introduces the state estimation strategy proposed for the nonlinear diffusion system and investigates the convergence problem. Simulation results obtained in the case of a steel rod are reported and discussed in Section 6 whereas Section 7 concludes the paper.

\section{Problem formulation}

Nonlinear diffusion equation describes a variety of nonlinear diffusion phenomena [35]. Hence, for the sake of simplicity, the following discussion is restricted to heat conduction transfer. The evolution of the temperature of a one dimensional rod, in the case of a temperaturedependent thermal conductivity, is described by the following nonlinear diffusion equation [11]

$\rho C_{p}(T(z, t)) \frac{\partial T(z, t)}{\partial t}=\frac{\partial}{\partial z}\left(k(T(z, t)) \frac{\partial T(z, t)}{\partial z}\right)$

with, without loss of generality, the following Dirichlet boundary conditions $(z \in \partial \Omega)$

$T(0, t)=T_{0}$

$T(l, t)=u(t)$

and the initial condition

$T(z, 0)=T_{\text {init }}(z)$

where $z \in \Omega=[0, l], \partial \Omega=\{0, l\}, z \in \Omega$ and $t \in$ $[0, \infty)$, are the spatial domain, spatial domain boundary, independent space and time variables, respectively. $T(z, t)$ is the state of the system (the rod temperature) that belongs to the Hilbert space $L^{2}(\Omega)\left(L^{2}(\Omega)\right.$ is the space of square-integrable functions on $\Omega) . \rho, C_{p}$ and $k$, that depend on the temperature of the $\operatorname{rod} T(z, t)$, are the density, heat capacity and thermal conductivity, respectively. The external manipulated heat source $u(t) \in L^{2}(\Omega, \Re)$ ( $\Re$ being the set of real numbers) is assumed to be a smooth function of the time. $T_{0}$ is a constant temperature and $T_{\text {init }}(z)$ is the initial spatial profile of the temperature.

The problem addressed in this work consists in estimating the whole state $T(z, t)$ from the available measurements. The choice of the sensing points will be discussed in Subsection 5.1.

The thermal diffusivity $\alpha$ being equal to

$\alpha=\frac{k(T(z, t))}{\rho C_{p}(T(z, t))}$

it must be noted that, in many practical cases, its variation with respect to temperature $T(z, t)$ is less important than that of the thermal conductivity $k(T(z, t))$. Consequently, assuming the thermal diffusivity $\alpha$ approximately constant is a physically reasonable approximation $[11,3]$. In this work, it is assumed that this assumption holds, which allows to derive an equivalent linear model of the nonlinear diffusion system (1)-(4) using the Kirchhoff tangent transformation [28,3] presented in the following section. 
Remark 1 In the formulated problem, a boundary control is considered but the development that follows can be applied in the case of distributed control.

\section{Kirchhoff tangent transformation}

A tangent transformation is a mathematical tool used to reduce a hard nonlinear PDE into a well-known and understood linear one, for instance into simple heat or wave equations [21]. Hence, the exact or the closed-form solution of the nonlinear PDE can be easily deduced from the solution of its equivalent linear one. Note that simple linear PDEs can be solved using some powerful techniques like separation of variables, eigenfunction expansions and integral transforms [11]. For a detailed discussion about some tangent transformations (Legendre, Cole-Hopf, Bäcklund and Laplace transformations) with illustrative examples, the reader can refer to the book by [21]. The Kirchhoff tangent transformation [3], used in this work, which is a particular case of the ColeHopf tangent transformation, is discussed at length by [28] and illustrated by interesting application examples.

As pointed out above, in many practical situations, the thermal diffusivity $\alpha$ can be assumed constant. Based on this reasonable assumption, the nonlinear diffusion system (1)-(4) can be linearized by the Kirchhoff tangent transformation $\varphi$ defined as follows

$$
\begin{aligned}
\varphi: L^{2}(\Omega) & \longrightarrow L^{2}(\Omega) \\
\theta(z, t) & \longmapsto T(z, t)=\varphi(\theta(z, t))
\end{aligned}
$$

where $\theta(z, t)$ is the new temperature variable depending on time and space, of the resulting equivalent linear diffusion model and $\varphi$ is a bijective mapping, that is, its inverse $\varphi^{-1}$ exists and is defined as follows [28].

The thermal conductivity is often available in the data literature as a nonlinear expression, for example a polynomial, with respect to temperature $T_{c}$ expressed in Celsius

$k_{a}\left(T_{c}\right)=a_{0}+a_{1} T_{c}+a_{2} T_{c}^{2}$

if the polynomial is of order 2 . It is also accompanied by a validity domain. It can be rewritten as

$k_{b}\left(T_{c}\right)=a_{0}\left(1+b_{1} T_{c}+b_{2} T_{c}^{2}\right)$

However, in the following, temperature $T$ will be expressed in Kelvin so that the previous expression becomes

$k_{c}(T)=c_{0}\left(1+c_{1} T+c_{2} T^{2}\right)$

Note that the heat conductivity could be any function such as

$k_{c}(T)=c_{0}\left(1+f_{c}(T)\right)$
In the literature, the Kirchhoff transformation that allows to transform the nonlinear diffusive equation into a linear equation is in general presented $[14,22]$ as

$$
\begin{aligned}
\theta(z, t) & =\varphi_{c}^{-1}(T(z, t)) \\
& =\frac{1}{k_{c}(0)} \int_{0}^{T(z, t)} k_{c}(s) d s
\end{aligned}
$$

however the correlation of conductivity is in general not valid at absolute zero temperature. Thus, it is more appropriate to define the Kirchhoff transformation as

$$
\begin{aligned}
\theta(z, t) & =\varphi_{c}^{-1}(T(z, t)) \\
& =T_{\text {ref }}+\frac{1}{k_{c}\left(T_{\text {ref }}\right)} \int_{T_{\text {ref }}}^{T(z, t)} k_{c}(s) d s
\end{aligned}
$$

where $T_{\text {ref }}$ is any temperature belonging to the validity domain of $k(T)$. Note that the previous definition differs slightly from [28] due to the introduction of $T_{\text {ref }}$, whereas [28] introduces a deviation variable $\tilde{T}=T-T_{\text {ref }}$ so that, with this notation, the thermal conductivity is

$k_{d}(\tilde{T})=d_{0}\left(1+f_{d}(\tilde{T})\right)$

and the Kirchhoff transformation writes as

$$
\begin{aligned}
\theta(z, t) & =\varphi_{d}^{-1}(\tilde{T}(z, t)) \\
& =\frac{1}{k_{d}(0)} \int_{0}^{\tilde{T}(z, t)} k_{d}(s) d s
\end{aligned}
$$

Indeed, Kirchhoff transformations (15) and (18) can be used equivalently, but eq.(18) will lead to simpler expressions. Consequently, we will retain the Kirchhoff transformation (18) in the following and omit the subscript "d".

Now, by using a Kirchhoff tangent transformation $\varphi$, the nonlinear diffusion system (1)-(4) is reduced to the following linear one

$\frac{\partial \theta(z, t)}{\partial t}=\alpha \frac{\partial^{2} \theta(z, t)}{\partial z^{2}}$

with associated boundary and initial conditions

$\theta(0, t)=\varphi^{-1}\left(\tilde{T}_{0}\right)$

$\theta(l, t)=\varphi^{-1}(\tilde{u}(t))$

$\theta(z, 0)=\varphi^{-1}\left(\tilde{T}_{\text {init }}(z)\right)$

In this work, a state estimation strategy for the nonlinear diffusion system (1)-(4) is proposed based on the use of the resulting equivalent linear diffusion model (19)-(22). The idea is to reconstruct the whole state of the nonlinear diffusion system $T(z, t)$ from the estimated state $\hat{\theta}(z, t)$ of the linear diffusion system using the Kirchhoff tangent transformation (7). 
Before presenting the proposed state estimation strategy, let us review briefly the state estimation theory of linear DPSs. A detailed discussion on the subject can be found in [6] while [12] gives a full review of the different linear observers proposed in the literature.

\section{State estimation for linear DPSs}

Let us consider a linear DPS described by an abstract formulation $[6,9]$

$$
\begin{aligned}
\dot{\theta}(t) & =\mathcal{A} \theta(t)+\mathcal{B} u(t) \\
y(t) & =\mathcal{C} \theta(t) \\
\theta(0) & =\theta_{\text {init }}
\end{aligned}
$$

where $\theta \in \mathcal{H}$ is the state of the linear DPS, $u(t) \in \mathcal{U}$ is the manipulated variable, $y(t) \in \mathcal{Y}$ is the output and $\theta_{0} \in \mathcal{H}$ is the initial condition. $\mathcal{H}, \mathcal{U}$ and $\mathcal{Y}$ are appropriate infinite dimensional spaces [9]. $A \in \mathcal{L}(\mathcal{H})$, $B \in \mathcal{L}(\mathcal{U}, \mathcal{H})$ and $C \in \mathcal{L}(\mathcal{H}, \mathcal{Y})$ are linear operators. $\mathcal{L}$ is the space of linear operators.

Assume that the linear DPS (23)-(25) is observable, that is, the state $\theta(z, t)$ can be reconstructed from the available measurements (24). In this case, the observer takes the following form

$$
\begin{aligned}
& \dot{\hat{\theta}}(t)=\mathcal{A} \hat{\theta}(t)+\mathcal{B} u(t)+\mathcal{L}(y(t)-\mathcal{C} \hat{\theta}(t)) \\
& \hat{\theta}(0)=\hat{\theta}_{\text {init }}
\end{aligned}
$$

where $\hat{\theta} \in \mathcal{H}$ is the estimate of the state $\theta . \mathcal{L}$ is the observer gain operator which is designed so that the operator $\mathcal{A}-\mathcal{L} \mathcal{C}$ generates an exponential stable $C_{0^{-}}$ semigroup $S(t)$, that is, $\|S(t)\|_{\mathcal{H}} \leq M e^{-\alpha t}$ with $M \geq 1$ and $\alpha>0$ [10]. This condition ensures that the estimation error defined as follows (Pourquoi est-ce nécessaire que $M>1$ ? J'aurais pensé simplement $M>0$. Ce n'est pas très important, mais je me faisais la réflexion.)

$$
\begin{aligned}
\varepsilon(t) & =\theta(t)-\hat{\theta}(t) \\
& =S(t) \varepsilon(0)
\end{aligned}
$$

tends exponentially to zero as $t \rightarrow \infty[6]$, i.e.,

$$
\|\varepsilon(t)\|_{\mathcal{H}} \leq M\|\varepsilon(0)\|_{\mathcal{H}} e^{-\alpha t}
$$

A complete review of different methods used to design an observer for a linear DPS can be found in [12] and some application examples are reported in [24] and [6].

\section{State estimation strategy for the nonlinear diffusion system}

State estimation theory for a nonlinear DPS remains a challenging field with many interesting open issues among which the design of the observer occupies an important place. In this section, a state estimation strategy is developed for the nonlinear diffusion system (1)(4).

\subsection{Observability analysis}

The observability analysis of the system is a primary issue for state estimation. This fundamental control property, in the case of DPSs, can be ensured by an appropriate choice of the location of the sensing points $[24,33]$. Note that the observability analysis of linear DPSs is an established and developed area with many interesting results $[24,6]$ compared to nonlinear DPSs for which the question is very complex. In the proposed approach, the design of the observer for the nonlinear diffusion system (1)-(4) is reduced to the design of an observer for the resulting linear diffusion system (19)(22), therefore the observability condition concerns the resulting linear diffusion system rather than the nonlinear diffusion one. Thus, in the following, it is assumed that the measurements (24) of the linear DPS (19)(22) are defined so that the observability condition is met. Hence, using (18), the measurements (24) can be expressed according to $T(z, t)$ as follows

$y(t)=\mathcal{C} \varphi^{-1}(\tilde{T}(z, t))$

which implies that the locations of the sensing points of the nonlinear diffusion system (1)-(4) are the same as those of linear diffusion (19)-(22).

\subsection{Proposed state estimation strategy}

The idea consists in taking advantage of the state estimation theory of linear DPS, which has reached a high degree of maturity with sound contributions [12]. The proposed state estimation approach consists in the following steps:

- linearize the nonlinear diffusion system (1)-(4) using the Kirchhoff tangent transformation (7),

- design an observer for the resulting linear diffusion system (19)-(22),

- reconstruct the estimated state, denoted $\hat{T}(z, t)$, of the nonlinear diffusion system (1)-(4) from the estimated state $\hat{\theta}(z, t)$ of the equivalent linear diffusion 
system (19)-(22) using the Kirchhoff tangent transformation (7), that is,

$$
\hat{\tilde{T}}(z, t)=\varphi(\hat{\theta}(z, t))
$$

The structure of the proposed observer is given by the following proposition.

Proposition 1 The estimated state $\hat{T}(z, t)$ of the nonlinear diffusion system (1)-(4) can be reconstructed, based on the resulting linear diffusion system (19)-(22), by the following observer

$$
\begin{aligned}
\frac{\partial \hat{\theta}(z, t)}{\partial t} & =\alpha \frac{\partial^{2} \hat{\theta}(z, t)}{\partial z^{2}}+\mathcal{L}(y(t)-\mathcal{C} \hat{\theta}(z, t)) \\
y(t) & =\mathcal{C} \varphi^{-1}(\tilde{T}(z, t))
\end{aligned}
$$

with the boundary and initial conditions (20)-(22). T( $z, t)$ is the measured temperature. The estimated temperature results as

$\hat{T}(z, t)=\varphi(\hat{\theta}(z, t))+T_{\text {ref }}$

Proof Recall that the operator $\mathcal{L}$ is designed based on the state estimation theory of linear DPSs so that the following operator

$\hat{\mathcal{A}}=\alpha \frac{\partial^{2}}{\partial z^{2}}-\mathcal{L C}$

generates an exponential stable $C_{0}$-semigroup so that

$\lim _{t \rightarrow \infty}(y(t)-\mathcal{C} \hat{\theta}(z, t))=\lim _{t \rightarrow \infty} \mathcal{C}(\theta(z, t)-\hat{\theta}(z, t))=0$

and since the operator $\mathcal{C}$ is linear, it follows that the state estimation error of the linear diffusion system (19)-(22), i.e., $\varepsilon(t)=\theta(z, t)-\hat{\theta}(z, t)$ verifies

$\lim _{t \rightarrow \infty} \varepsilon(t)=\lim _{t \rightarrow \infty}(\theta(z, t)-\hat{\theta}(z, t))=0$

hence

$\lim _{t \rightarrow \infty} \varphi^{-1}(\tilde{T}(z, t))=\lim _{t \rightarrow \infty} \varphi^{-1}(\hat{\tilde{T}}(z, t))$

and since $\varphi$ is a bijective mapping, it follows that

$\lim _{t \rightarrow \infty} \tilde{T}(z, t)=\lim _{t \rightarrow \infty} \hat{\tilde{T}}(z, t)$

or

$\lim _{t \rightarrow \infty} T(z, t)=\lim _{t \rightarrow \infty} \hat{T}(z, t)$

or equivalently

$\lim _{t \rightarrow \infty}(T(z, t)-\hat{T}(z, t))=0$

therefore, the state estimation error of the nonlinear diffusion system $e(t)=T(z, t)-\hat{T}(z, t)$ converges exponentially to zero as $t \rightarrow \infty$.

The state estimation strategy proposed for the nonlinear diffusion system is depicted in Figure 1 where $v$ represents the measurement noise.

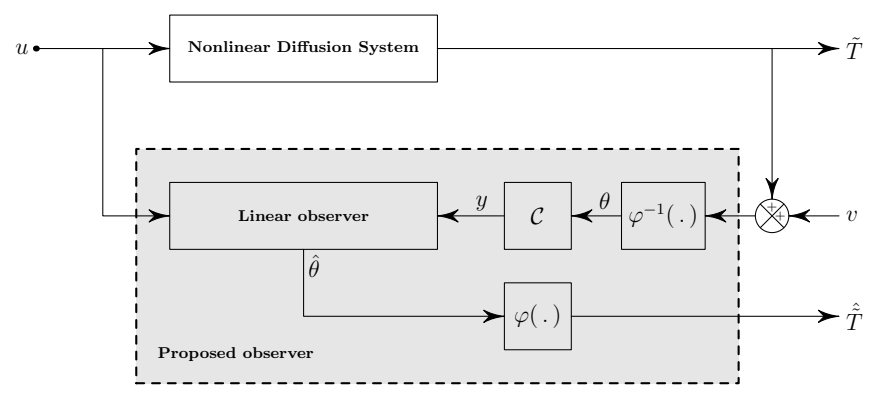

Fig. 1: Structure of the proposed observer for the nonlinear diffusion system.

\section{Application example}

To show the effectiveness of the proposed state estimation strategy for the nonlinear diffusion system, a steel rod of length $l=0.25 \mathrm{~m}$ is considered as an application example. The thermo-physical properties of carbon and low-alloyed steels are [27]:

$$
\begin{aligned}
\rho & =7820\left[\mathrm{~kg} \cdot \mathrm{m}^{-3}\right] \\
C_{p}\left(T_{c}\right) & =422+0.931 T_{c}-2.14 \times 10^{-3} T_{c}^{2} \\
& +2.64 \times 10^{-6} T_{c}^{3}\left[\mathrm{~J} \cdot \mathrm{kg}^{-1} \cdot \mathrm{K}^{-1}\right] ; 0 \leq T_{c} \leq 650 \\
k\left(T_{c}\right) & =52.1-0.0159 T_{c} \\
& -1.86 \times 10^{-5} T_{c}^{2}\left[\mathrm{~W} \cdot \mathrm{m}^{-1} \cdot \mathrm{K}^{-1}\right] ; 0 \leq T_{c} \leq 650
\end{aligned}
$$

where $T_{c}$ is the temperature in Celsius. Under the polynomial form (45)

$k(\tilde{T})=d_{0}\left(1+d_{1} \tilde{T}+d_{2} \tilde{T}^{2}\right)$

the coefficients of the heat conductivity are $d_{0}=44.968$, $d_{1}=-6.2244 \times 10^{-4}, d_{2}=-4.1363 \times 10^{-7}$ when the deviation temperature $\tilde{T}=T-T_{\text {ref }}$ is used. The reference temperature $T_{r e f}$ is taken as the mean of the validity domain for the heat conductivity, i.e. $T_{r e f}=598.15 \mathrm{~K}$.

$T(z, t)$ is the temperature (in $\mathrm{K}$ ) of the steel rod considered at any position and any time, i.e. $T(z, t)$. The dynamic behavior of the steel rod temperature is described by the nonlinear diffusion equation (1). The evolution of the non-constant thermal diffusivity $\alpha(T)$ as a function of the temperature $T$, between $273.15 \mathrm{~K}$ and $923.15 \mathrm{~K}$, is given by Figure 2, which shows a low variation of $\alpha(T)$. Therefore, $\alpha$ can be assumed constant. Thus, for the equivalent linear diffusion system, the constant thermal diffusivity $\alpha$ is taken equal to the mean value of the non-constant $\alpha(T)$, hence $\alpha=$ $9.85 \times 10^{-6} \mathrm{~m}^{2} \cdot \mathrm{s}^{-1}$.

To simplify the expression of $\theta$, Kirchoff transformation (18) is preferred with regard to (15). According 


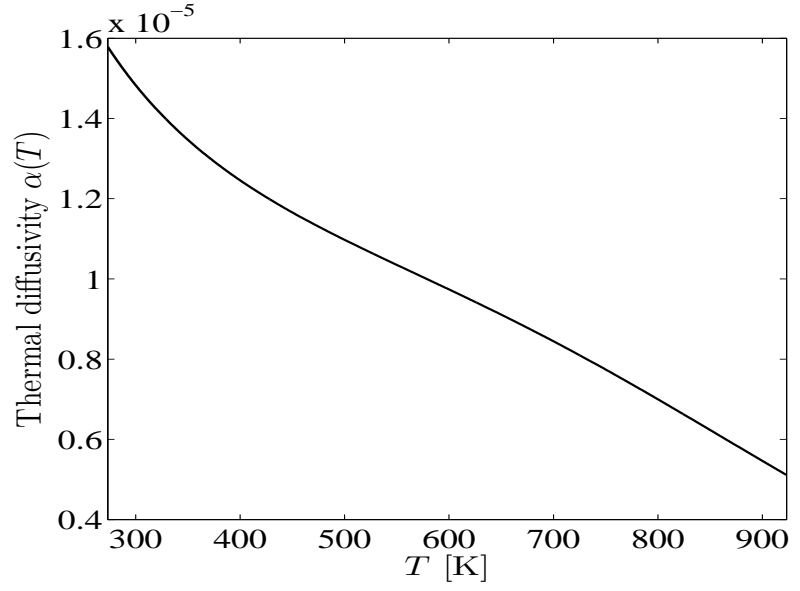

Fig. 2: Variation of the steel thermal diffusivity $\alpha(T)$ as a function of the temperature $T$.

to the inverse Kirchhoff tangent transformation (18), the state of the equivalent linear system $\theta(z, t)$ and the state of the nonlinear diffusion system $T(z, t)$ with $\tilde{T}(z, t)=T(z, t)-T_{\text {ref }}$ are linked by the following relation

$$
\begin{aligned}
\theta(z, t) & =\varphi^{-1}(\tilde{T}(z, t)) \\
& =\tilde{T}(z, t)-3.1122 \times 10^{-4} \tilde{T}^{2}(z, t) \\
& -1.3788 \times 10^{-7} \tilde{T}^{3}(z, t)
\end{aligned}
$$

Clearly, the nonlinearity of the application $\varphi$ or equivalently $\varphi^{-1}$ depends on the order of magnitude of the coefficients of the first and second order terms of the expression of $k(\tilde{T})$ which intervene indirectly in (47). In the particular case (47), this nonlinearity is extremely small.

The performance of the proposed observer is evaluated through numerical simulation runs. Both system and observer are implemented using the method of lines [29]. The convergence of proposed observer is demonstrated using the $L^{2}$-norm of the estimation error defined as follows

$\|e(t)\|_{L^{2}(\Omega)}^{2}=\int_{0}^{l}[T(z, t)-\hat{T}(z, t)]^{2} d z$

In the following, two practical cases are studied.

\subsection{Uniform distributed noisy sensing}

In this case, it is assumed that the system is autonomous (without external heat source $u(t)$ ), i.e., it is subject to the initial spatial temperature profile $T(z, 0)$. The problem consists in reconstructing the whole state $T(z, t)$ from uniformly distributed measurements corrupted with
Gaussian white noise signal $v(z, t)$ of standard deviation $\sigma=50 \mathrm{~K}$.

The model is given as follows

$$
\begin{aligned}
\rho C_{p}(T(z, t)) \frac{\partial T(z, t)}{\partial t} & =\frac{\partial}{\partial z}\left(k(T(z, t)) \frac{\partial T(z, t)}{\partial z}\right) \\
T(0, t) & =450[\mathrm{~K}] \\
T(l, t) & =900[\mathrm{~K}] \\
T(z, 0) & =450(4 z+1)[\mathrm{K}]
\end{aligned}
$$

The Kirchhoff tangent transformation yields the following linear diffusion system

$$
\begin{aligned}
\frac{\partial \theta(z, t)}{\partial t} & =\alpha \frac{\partial^{2} \theta(z, t)}{\partial z^{2}} \\
\theta(0, t) & =\varphi^{-1}(\tilde{T}(0, t)) \\
\theta(l, t) & =\varphi^{-1}(\tilde{T}(l, t)) \\
\theta(z, 0) & =\varphi^{-1}(\tilde{T}(z, 0))
\end{aligned}
$$

which can be filtered by the following observer [20]

$$
\begin{aligned}
\frac{\partial \hat{\theta}(z, t)}{\partial t}= & \alpha \frac{\partial^{2} \hat{\theta}(z, t)}{\partial z^{2}} \\
& +6 \sin (\pi z) \int_{0}^{l}(y(z, t)-\hat{\theta}(z, t)) d z
\end{aligned}
$$

where

$y(z, t)=\varphi^{-1}\left(\tilde{T}_{n}(z, t)\right)$

and $\tilde{T}_{n}(z, t)$ is the measurement.

Let us express $y(z, t)$, according to (46), as a function of the noisy state denoted $T_{n}(z, t)$ as follows

$$
\begin{aligned}
y(z, t) & =\tilde{T}_{n}(z, t)-3.1122 \times 10^{-4} \tilde{T}_{n}^{2}(z, t) \\
& -1.3788 \times 10^{-7} \tilde{T}_{n}^{3}(z, t)
\end{aligned}
$$

with $T_{n}(z, t)=T(z, t)+v(z, t)$, and $\tilde{T}_{n}=T_{n}-T_{\text {ref }}$. $v(z, t)$ is a white noise. The proposed observer takes the following form

$$
\begin{aligned}
\frac{\partial \hat{\theta}(z, t)}{\partial t}= & \alpha \frac{\partial^{2} \hat{\theta}(z, t)}{\partial z^{2}} \\
& +6 \sin (\pi z) \int_{0}^{l}(y(z, t)-\hat{\theta}(z, t)) d z \\
\hat{\theta}(z, 0)= & \theta_{\text {init }} \\
\hat{\tilde{T}}(z, t)= & \varphi(\hat{\theta}(z, t))
\end{aligned}
$$

with the boundary conditions (54) and (55).

The simulation results obtained, by taking $\hat{\theta}_{\text {init }}=$ $\varphi^{-1}(\tilde{T}(z, 0))+50[\mathrm{~K}]$, i.e. an initial error of $50 \mathrm{~K}$, are given by Figures $3-5$. Figure 3 clearly shows that the estimation error, which is bounded, converges towards 


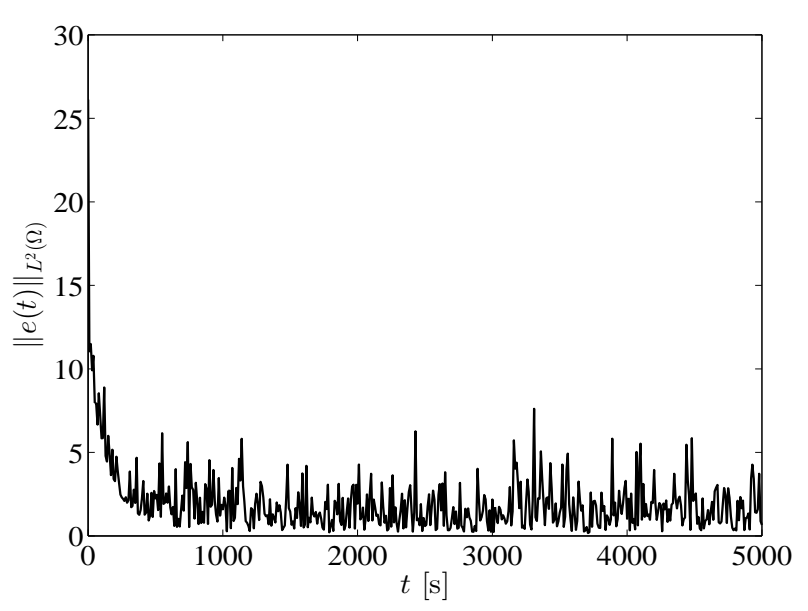

Fig. 3: Uniformly distributed sensing with noisy measurements: evolution of the $L^{2}$-norm of the estimation error $e(t)=T(z, t)-\hat{T}(z, t)$.

zero without reaching it. This is expected because, with noisy measurements, achieving a zero estimation error is not possible.

The ability of the proposed observer in reconstructing the whole state $T(z, t)$ despite the effects of measurement noises $v(z, t)$ is corroborated by Figures 4 and 5 that give the temporal and spatial profiles, respectively. From these Figures, it follows that the proposed observer enables to provide the estimated state of the nonlinear diffusion system.

\subsection{Punctual boundary sensing}

In this case, it is assumed that the steel rod is heated by an external heat source at the extremity $l$ so that $u(t)=$ $100\left(1-e^{-1 / 3500 t}\right)+300[\mathrm{~K}]$, applied at the right-hand end boundary $z=l$, and the objective is to estimate the whole state $T(z, t)$ from the measurement of the temperature at $z=l$.

The model is given as follows

$$
\begin{aligned}
\rho C_{p}(T(z, t)) \frac{\partial T(z, t)}{\partial t} & =\frac{\partial}{\partial z}\left(k(T(z, t)) \frac{\partial T(z, t)}{\partial z}\right) \\
\left.\frac{\partial T(z, t)}{\partial z}\right|_{z=0} & =-q T(0, t)\left[\mathrm{K} \cdot \mathrm{m}^{-1}\right] \\
T(l, t) & =100\left(1-e^{-1 / 3500 t}\right)+300[\mathrm{~K}] \\
T(z, 0) & =500 z^{2}-27.5 z+275[\mathrm{~K}]
\end{aligned}
$$

with $q=0.1\left[\mathrm{~m}^{-1}\right]$.
(Pourquoi a-t'on $u(t)=T(l, t)$ : voir ce qui précède dans cette section. L'équation (62) correspond à (25) avec $\mathcal{B}=0$. Je ne vois pas de terme en $u$. L'équation (62) correspond d'ailleurs à l'équation (66). Il faut vérifier ces points pour que ce soit cohérent. Ne faudrait-il pas pour avoir cette cohérence écrire l'équation (64) comme $T(l, t)=u(t)$ au lieu de la forme numérique actuelle pour bien faire apparaître le lien (je pense que c'est ce qu'il faut faire) ? Par contre, écrire $T(l, t)=u(t)$ (condition de Dirichlet) est vraiment une vision très mathématique, pas physique, car $u$ correspond à un flux de chaleur (condition de Neumann) et $T$ est une temp érature, donc les deux n'ont pas la même dimen-sion. D'un vrai point de vue physique, on devrait écrire $u(t)=-k(\partial T / \partial z)_{z=l}$ ce qui nous entraîne un peu trop loin à quelques jours de l'envoi de l'article mais aurait pu très facilement être pis en compte.)

The Kirchhoff tangent transformation (7) yields the following linear model

$$
\begin{aligned}
\frac{\partial \theta(z, t)}{\partial t} & =\alpha \frac{\partial^{2} \theta(z, t)}{\partial z^{2}} \\
\left.\frac{\partial \theta(z, t)}{\partial z}\right|_{z=0} & =-q \varphi(\theta(0, t)) \\
\theta(l, t) & =\varphi^{-1}(\tilde{u}(t)) \\
\theta(z, 0) & =\varphi^{-1}(\tilde{T}(z, 0))
\end{aligned}
$$

with $\tilde{u}=u-T_{\text {ref }}$. Note that, though the nonlinear diffusion equation is linearized, a nonlinearity, that is, $\varphi(\theta(0, t))$, appears in the right-side hand of the resulting boundary condition (67). The importance of the nonlinear character can be evaluated from the expression of $\theta(z, t)$ in equation (47). Presently

$\tilde{T}(0, t)=\varphi(\theta(0, t)) \approx \theta(0, t)$

The estimated state $\hat{\theta}(z, t)$ can be provided using a backstepping observer of the following form [26]

$$
\begin{aligned}
\frac{\partial \hat{\theta}(z, t)}{\partial t}= & \alpha \frac{\partial^{2} \hat{\theta}(z, t)}{\partial z^{2}} \\
& -\alpha q e^{q(1-z)}\left(y(t)-\frac{\partial \hat{\theta}(l, t)}{\partial z}\right) \\
\left.\frac{\partial \hat{\theta}(z, t)}{\partial z}\right|_{z=0}= & -q \hat{\theta}(0, t) \\
\hat{\theta}(l, t)= & \varphi^{-1}(\tilde{u}(t)) \\
\hat{\theta}(z, 0)= & \varphi^{-1}(\tilde{T}(z, 0))
\end{aligned}
$$

where $y(t)$ is proportional to the heat flux at $z=l$ given as follows

$y(t)=\left.\frac{\partial \varphi^{-1}(\tilde{T}(z, t))}{\partial z}\right|_{z=l}$ 

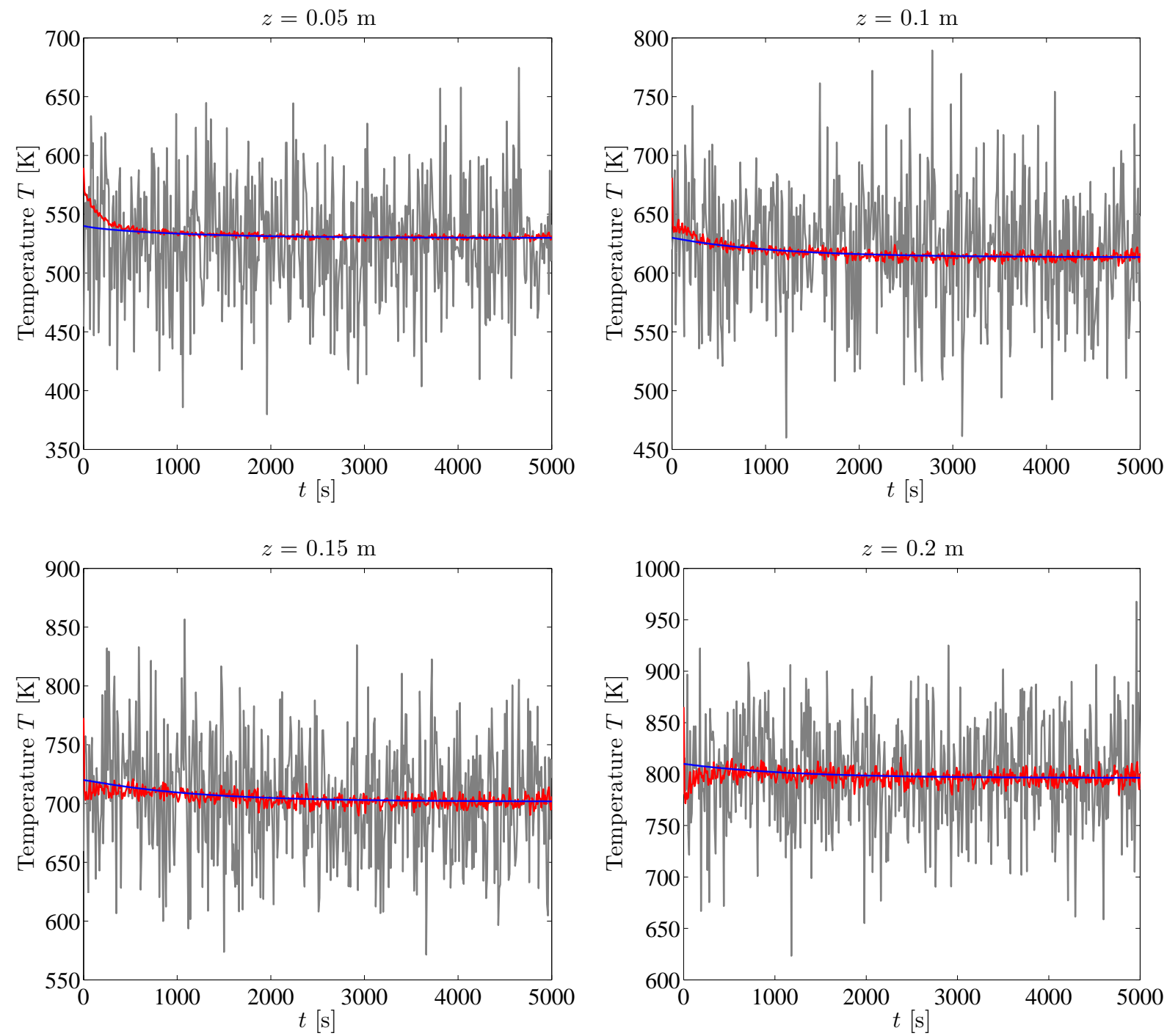

Fig. 4: Uniformly distributed sensing with noisy measurements: temporal profiles. Theoretical state $T(z, t)$ (blue), measured state $T_{n}(z, t)$ (gray) and estimated state $\hat{T}(z, t)$ (red).

Let us express the output (75) as a function of the measurement $\tilde{T}(z, t)$. Taking into account $(46)$, it results that

$$
\begin{aligned}
y(t)= & \left.\left.\frac{\partial \theta(z, t)}{\partial \tilde{T}(z, t)}\right|_{z=l} \frac{\partial \tilde{T}(z, t)}{\partial z}\right|_{z=l} \\
= & \left.\left.\frac{\partial \varphi^{-1}(\tilde{T}(z, t))}{\partial \tilde{T}(z, t)}\right|_{z=l} \frac{\partial \tilde{T}(z, t)}{\partial z}\right|_{z=l} \\
= & \left(1-6.224 \times 10^{-4} \tilde{T}(l, t)\right. \\
& \left.-4.1363 \times 10^{-9} \tilde{T}^{2}(l, t)\right)\left.\frac{\partial \tilde{T}(l, t)}{\partial z}\right|_{z=l}
\end{aligned}
$$

hence the following proposed observer results

$$
\begin{aligned}
\frac{\partial \hat{\theta}(z, t)}{\partial t}= & \alpha \frac{\partial^{2} \hat{\theta}(z, t)}{\partial z^{2}} \\
& -\alpha q e^{q(1-z)}\left(y(t)-\frac{\partial \hat{\theta}(l, t)}{\partial z}\right) \\
\left.\frac{\partial \hat{\theta}(z, t)}{\partial z}\right|_{z=0} & =-q \hat{\theta}(0, t) \\
\hat{\theta}(l, t)= & \varphi^{-1}(\tilde{u}(t)) \\
\hat{\theta}(z, 0)= & \hat{\theta}_{\text {init }} \\
\hat{\tilde{T}}(z, t)= & \varphi(\hat{\theta}(z, t))
\end{aligned}
$$

The performance of the proposed observer in retrieving the state $T(z, t)$, by assuming the initial profile 

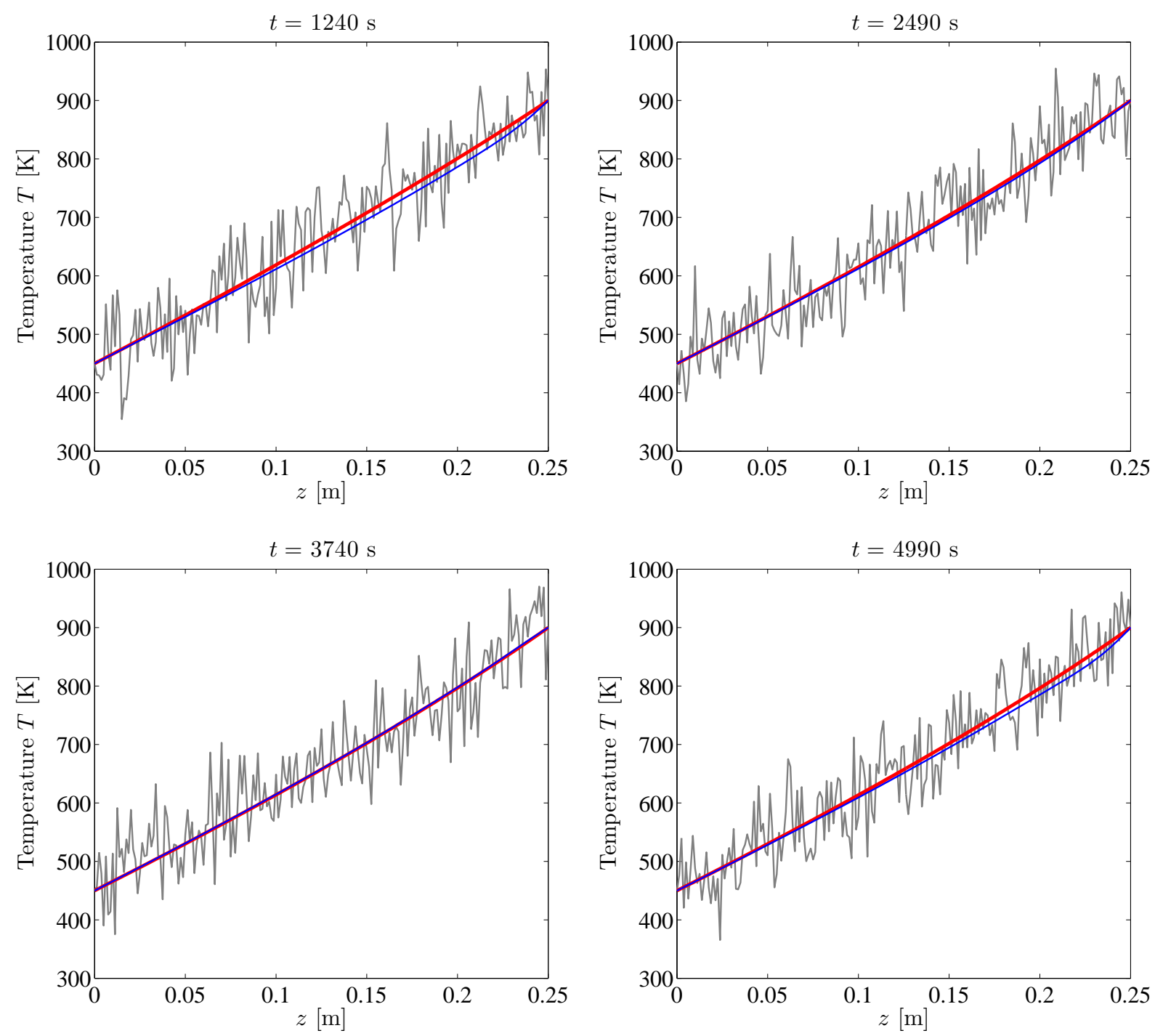

Fig. 5: Uniformly distributed sensing with noisy measurements: spatial profiles. Theoretical state $T(z, t)$ (blue), measured state $T_{n}(z, t)$ (gray) and estimated state $\hat{T}(z, t)$ (red).

$\hat{\theta}_{\text {init }}=\varphi^{-1}(\tilde{T}(z, 0))+150[\mathrm{~K}]$, is shown by Figures $6-8$. The obtained simulation results show the convergence of the estimated state $\hat{T}(z, t)$ towards the actual state $T(z, t)$, that is, the estimation state error tends to zero as $t \rightarrow \infty$ (Fig. 6) and both temporal and spatial profiles are accurately reconstructed (Figs. 7-8).

In the second simulation run, the performance of the proposed observer is evaluated again for a nonmonotonic smooth external heat source $u(t)$ defined as a series of increasing and decreasing exponential signals

$$
u(t)= \begin{cases}300+100\left(1-e^{-t / 3500}\right) & t<t_{1} \\ 300+100 e^{-\left(t-t_{1}\right) / 3500} & t_{1} \leq t \leq t_{2} \\ 300+100\left(1-e^{-\left(t-t_{2}\right) / 3500}\right) & t>t_{2}\end{cases}
$$

The evolution of the theoretical test signal $u(t)$ is depicted in Figure 9. The obtained results given by Figure 10 show the good agreement between the estimate $\hat{T}(z, t)$ and the theoretical $T(z, t)$ temperatures. This simulation run demonstrates the ability of the proposed observer in reconstructing the whole state from the boundary measurement.

On a utilisé le mot "non-monotonic" (croissant et d écroissant, c'est-à-dire sa dérivée change de signe) pour qualifier l'évolution du signal test utilisé. Je ne sais pas s'il y a mieux comme qualificatif. J'ai rajouté "as a series of increasing and decreasing exponential signals" 


\section{Conclusion}

In this paper, an observer is proposed to reconstruct the state of a nonlinear diffusion system from the available measurements. The proposed observer is developed by exploiting the well established state estimation theory of linear DPSs. Thus, instead of designing the observer for the nonlinear diffusion system, which is a difficult task, it is proposed to use the Kirchhoff tangent transformation to linearize the nonlinear diffusion system and an observer is designed for the obtained equivalent linear diffusion system in the framework of the state estimation theory of linear DPSs. The estimated state of the nonlinear diffusion system is then retrieved from the estimated state of the equivalent linear diffusion model using the Kirchhoff tangent transformation. The convergence of the proposed observer is demonstrated based on the fact that the Kirchhoff tangent transformation is a bijective mapping. The performance of the proposed observer in reconstructing the whole state of the nonlinear diffusion system is demonstrated by the obtained results concerning a heated steel rod taken as an application example.

Note that the proposed approach presents remarkable advantages, it allows to take full advantage from the state estimation theory of linear DPSs. In addition, the location of the sensors that ensures the observability property deals with the linear diffusion system instead of the nonlinear diffusion one. This allows, also, to avoid an acute problem encountered with nonlinear DPSs.

The success of the use of the Kirchhoff tangent transformation suggests to consider other tangent transformations to linearize some classes of nonlinear DPSs, which is under investigation by the authors.

\section{References}

1. S. Afshar, K. Morris, and A. Khajepour. Comparison of different observer designs for heat equation. In the Proceedings of the 54th IEEE Annual Conference on the Decision and Control (CDC), Osaka, Japan, pages 1136-1141, December 15-18, 2015.

2. M. Bitzer and M. Zeitz. Design of a nonlinear distributed parameter observer for a pressure swing adsorption plant. Journal of Process Control, 12(4):533-543, 2002.

3. H.S. Carslaw and J.C. Jaeger. Conduction of Heat in Solids. Oxford University Press, Oxford, UK, Second edition, 1959.

4. P.D. Christofides. Nonlinear and robust control of PDE systems: methods and applications to transport-reaction processes. Birkhäuser, Boston, 2001.

5. R. Curtain. A survey of infinite-dimensional filtering. SIAM Review, 17(3):395-411, 1975.
6. R.F. Curtain and H. Zwart. An Introduction to InfiniteDimensional Linear Systems Theory. Springer, New York, 1995.

7. A. El Jai and M. Amouroux. Sensors and observers in distributed parameter systems. International Journal of Control, 47(1):333-347, 1988.

8. F. Eleiwi and T. M. Laleg-Kirati. Nonlinear observerbased Lyapunov boundary control of distributed heat transfer mechanisms for membrane distillation plant. Journal of Process Control, 47:78-86, 2016.

9. Z. Emirsjlow and S. Townley. From PDEs with boundary control to the abstract state equation with an unbounded input operator: A tutorial. European Journal of Control, 6(1):27-49, 2000.

10. K.-J. Engel and R. Nagel. A Short Course on Operator Semigroups. Springer, 2006.

11. S.J. Farlow. Partial Differential Equations for Scientists and Engineers. Dover Publications, New York, USA, 1993.

12. Z. Hidayat, R. Babusška, B. De Schutter, and A. Núñez. Observers for linear distributed parameter systems: A survey. In the Proceedings of the 2011 IEEE International Symposium on Robotics and Sensors Environments (ROSE 2011), Montreal, Canada, pages 166-171, September 17$18,2011$.

13. X. Hua, M. Mangold, A. Kienle, and E.-D. Gilles. State profile estimation of an autothermal periodic fixed-bed reactor. Chemical Engineering Science, 53(1):47-58, 1997.

14. S. Kim. A simple direct estimation of temperaturedependent thermal conductivity with kirchhoff transformation. International Communications in Heat and Mass Transfer, 28(4):537-544, 2001.

15. H.-X. Li and C. Qi. Modeling of distributed parameter systems for applications-a synthesized review from timespace separation. Journal of Process Control, 20(8):891901, 2010.

16. A. Maidi and J.P. Corriou. Boundary control of nonlinear distributed parameter systems by input-output linearization. In the Proceedings of the 18th IFAC World Congress, Milan, Italy, pages 10910-10915, August 28-September 02, 2011.

17. M. Mangold, G. Lauschke, J. Schaffner, M. Zeitz, and E.-D. Gilles. State and parameter estimation for adsorption columns by nonlinear distributed parameter state observers. Journal of Process Control, 4(3):163-172, 1994.

18. W. Marquardt and H. Auracher. An observer-based solution of inverse heat conduction problems. International Journal of Heat and Mass Transfer, 33(7):1545-1562, 1990.

19. J.S. Meditch. On state estimation for distributed parameter systems. Journal of the Franklin Institute, 290(1):49$59,1970$.

20. J.S. Meditch. Least-squares filtering and smoothing for linear distributed parameter systems. Automatica, $7(3): 315-322,1971$.

21. S.V. Meleshko. Methods for Constructing Exact Solutions of Partial Differential Equations. Mathematical and Analytical Techniques with Applications in Engineering. SpringerVerlag, New York, 2005.

22. M. Mierzwiczak, W. Chen, and Z.-J Fu. The singular bounadry method for steady-state nonlinear heat conduction problem with temperature-dependent thermal conductivity. International Journal of Heat and Mass Transfer, 91:205-217, 2015.

23. V. T. Nguyen, D. Georges, and G. Besançon. State and parameter estimation in 1-D hyperbolic PDEs based on an adjoint method. Automatica, 67:185-191, 2016.

24. W.H. Ray. Advanced Process Control. Butterworths, Boston, 1989. 
25. A. Singh and J. Hahn. Effect of finite-dimensional approximation on observability analysis of distributed parameter models. In the Proceedings of the 8th IFAC Symposium on Dynamics and Control of Processes Systems, Cancun, Mexico, pages 199-204, June 6-8, 2007.

26. A. Smyshlyaev and M. Krstic. Backstepping observers for a class of parabolic PDEs. Systems and Control Letters, 54(7):613-625, 2005.

27. J. Taler and P. Duda. Solving Direct and Inverse Heat Conduction Problems. Springer, Berlin, 2006.

28. P. Vadasz. Analytical solution to nonlinear thermal diffusion: Kirchhoff versus Cole-Hopf transformations. Journal of Heat Transfer, 132(12):121 302.1-121 302.6, 2010.

29. A. Vande Wouwer, P. Saucez, and W.E. Schiesser. Adaptive method of lines. CRC Press, 2001.

30. A. Vande Wouwer and M. Zeitz. State estimation in distributed parameter systems. In Encyclopedia of Life Support Systems (EOLSS): Control Systems, Robotics and Automation. EOLSS, Paris, France, 2003.

31. A. Vande Wouwer, M. Zeitz, N. Point, and M. Remy. Online implementation of a nonlinear distributed observer for a multizone furnace: Comparative study with a nonlinear filter. Control Engineering Practice, 1(6):947-955, 1993.

32. D. Varies, K.J. Kessman, and H. Zwart. A luenberger observer for an infinite dimensional bilinear system: A UV disinfection example. In the Proceedings of the 3rd IFAC Symposium on Systems Structures and Control, Foz do Iguassu, Brazil, 2007.

33. W. Waldraff, D. Douchain, S. Bourrel, and A. Magnus. On the use of observability measures for sensor location in tubular reactor. Journal of Process Control, 8(5-6):497$505,1998$.

34. J.-W. Wang and Y. Guo. Distributed-parameter Luenberger observer for semi-linear parabolic PDE systems with a mobile pointwise sensor. In the proceedings of the 35th Chinese Control Conference, Chengdu, China, pages 1136-1141, July 27-29 2016.

35. Z. Wu, J. Zhao, J. Yin, and H. Li. Nonlinear Diffusion Equations. World Scientific Publishing, London, 2001. 


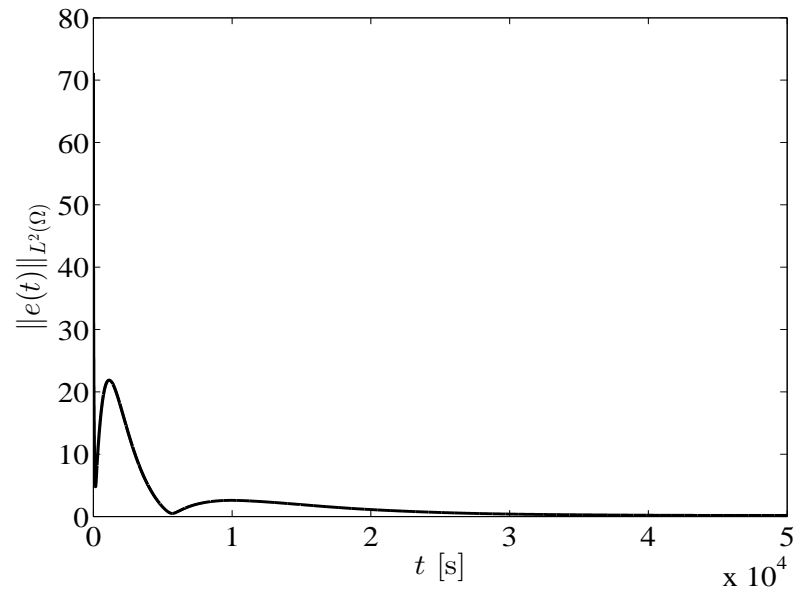

Fig. 6: Punctual boundary sensing: evolution of the $L^{2}$ norm of the estimation error $e(t)=T(z, t)-\hat{T}(z, t)$. 

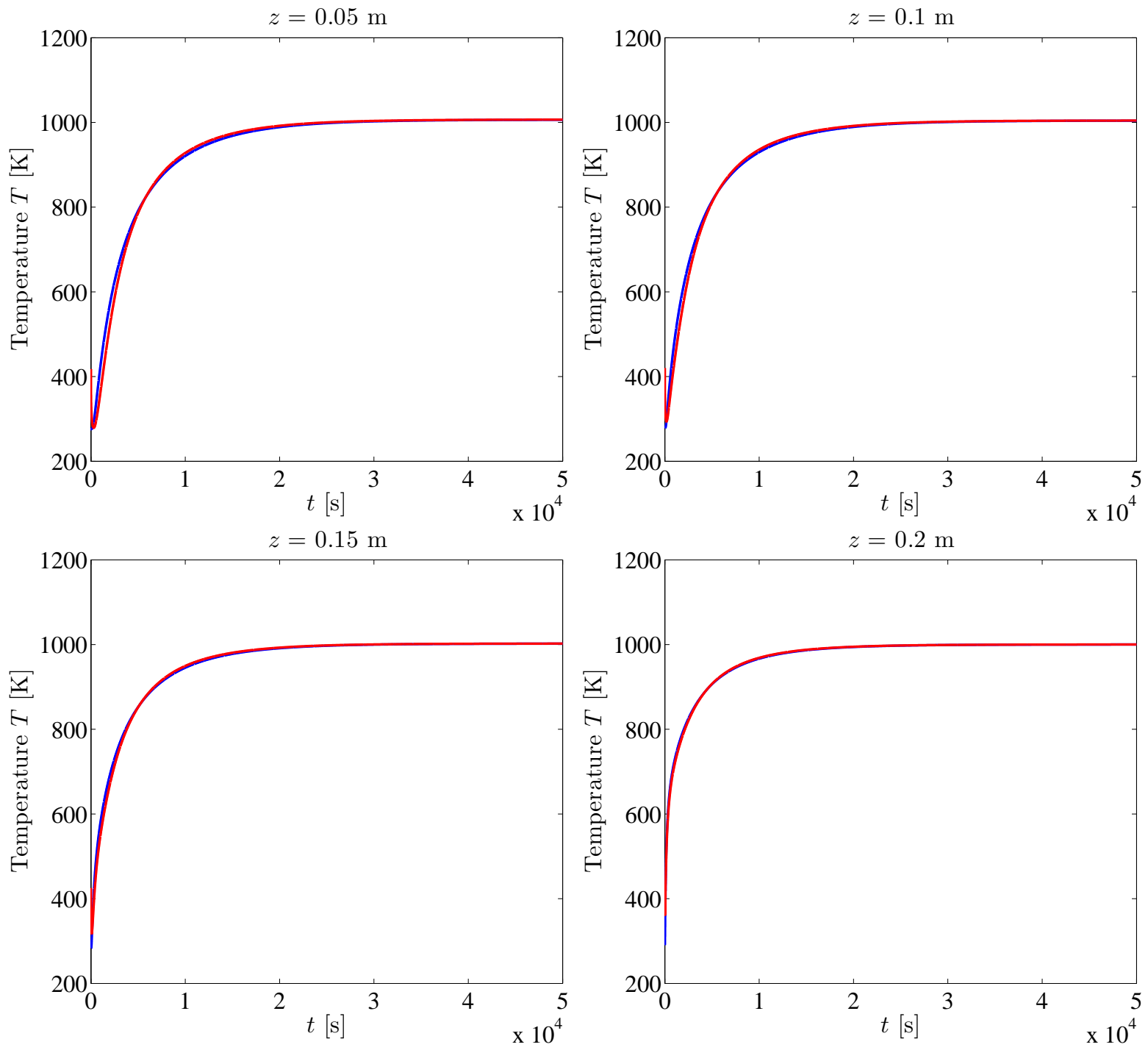

Fig. 7: Punctual boundary sensing: temporal profiles. Theoretical state $T(z, t)$ (blue) and estimated state $\hat{T}(z, t)$ (red). 

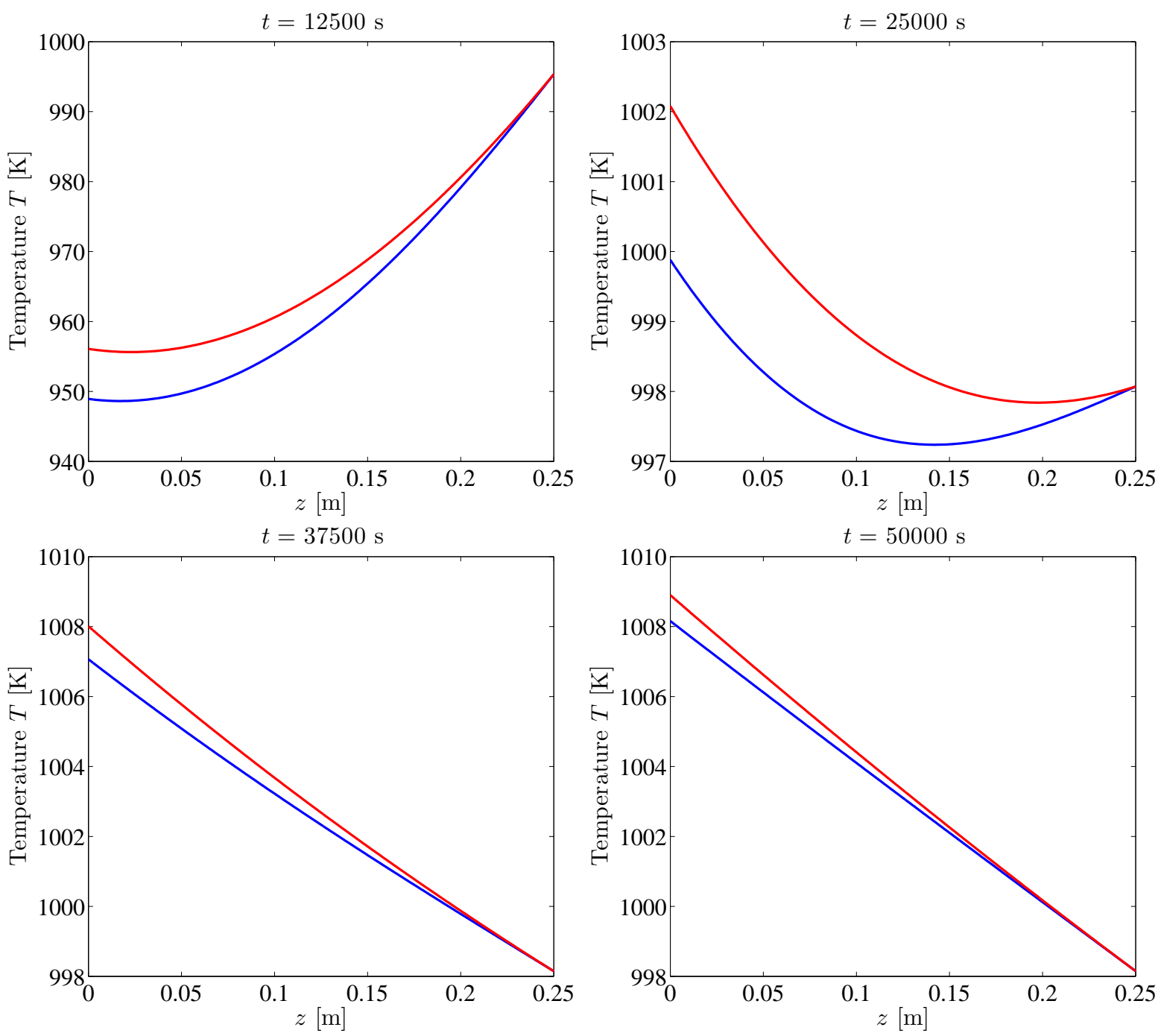

Fig. 8: Punctual boundary sensing: spatial profiles. Theoretical state $T(z, t)$ (blue) and estimated state $\hat{T}(z, t)$ (red). 


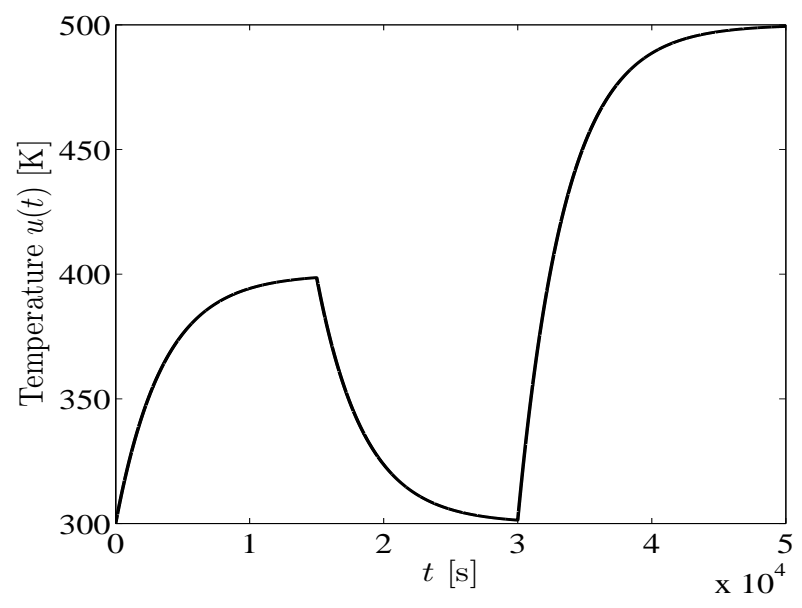

Fig. 9: Evolution of the test signal $u(t)$. 

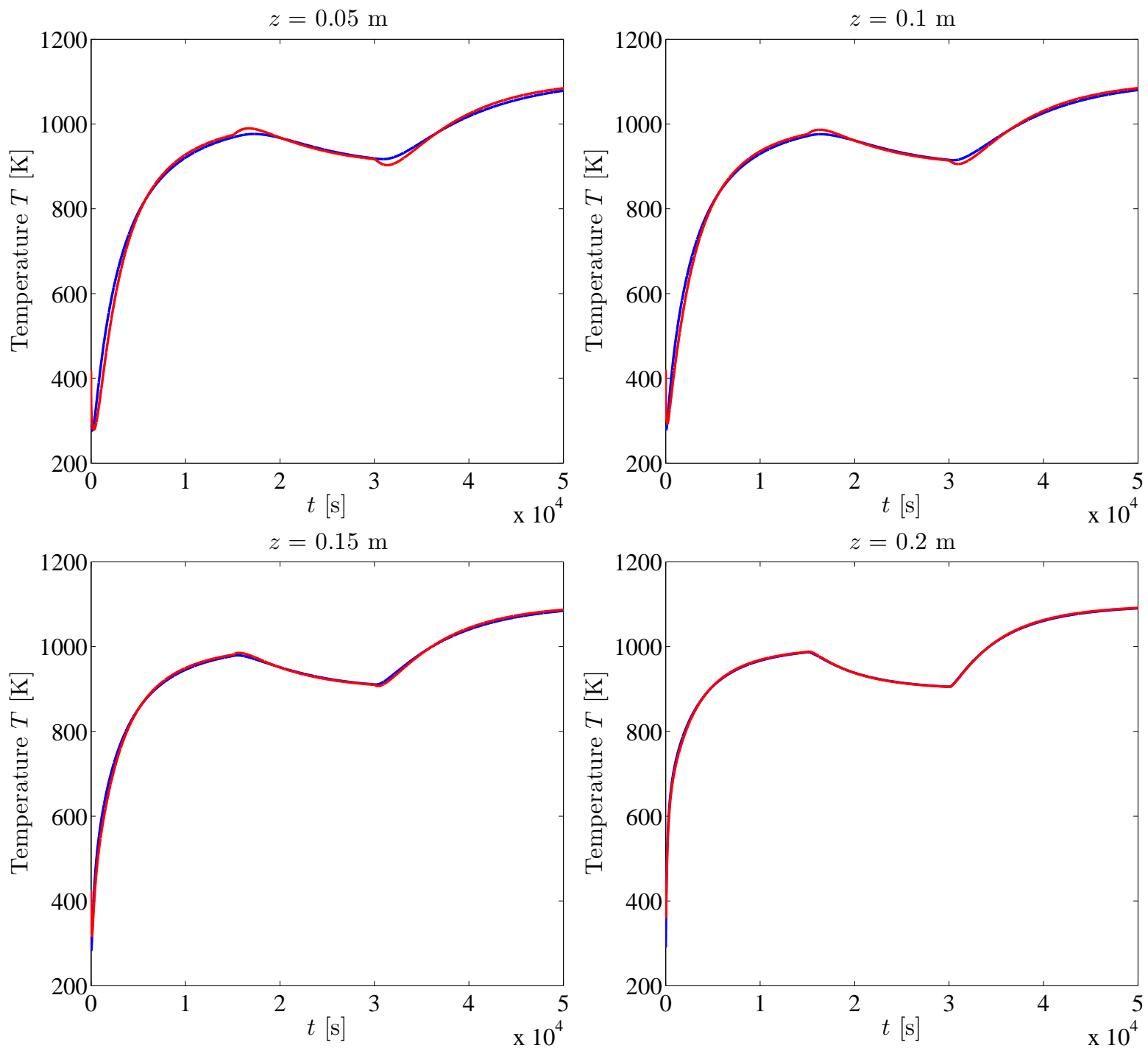

Fig. 10: Temporal profiles in the case of punctual boundary sensing for the test signal $u(t)$ given by (84). Theoretical state $T(z, t)$ (blue) and estimated state $\hat{T}(z, t)$ (red) in the case of . 